\title{
BMJ Open Endovascular treatment versus standard medical treatment for acute basilar artery occlusion: protocol for a systematic review and meta-analysis
}

To cite: Bai X, Zhang X, Li L, et al. Endovascular treatment versus standard medical treatment for acute basilar artery occlusion: protocol for a systematic review and meta-analysis. BMJ Open 2020;10:e040415. doi:10.1136/ bmjopen-2020-040415

- Prepublication history and additional material for this paper are available online. To view these files, please visit the journal online (http://dx.doi. org/10.1136/bmjopen-2020040415).

Received 13 May 2020 Revised 12 October 2020 Accepted 28 October 2020

Check for updates

(C) Author(s) (or their employer(s)) 2020. Re-use permitted under CC BY-NC. No commercial re-use. See rights and permissions. Published by BMJ.

For numbered affiliations see end of article.

Correspondence to

Dr Liqun Jiao; liqunjiao@sina.cn

\section{ABSTRACT}

Introduction Acute basilar artery occlusion (BAO)

can cause posterior circulation stroke. There are

two predominant therapies for BAO: standard

medical treatment (SMT) and SMT plus endovascular thrombectomy (EVT). However, a conclusive systematic comparison of the safety and efficacy of SMT and those of SMT plus EVT for the treatment of BAO is lacking. Thus, a systematic review and meta-analysis is needed to evaluate the safety and efficacy of SMT and SMT plus EVT for the treatment of BAO.

Methods and analysis This protocol is drafted referring to the Preferred Reporting Items for Systematic Reviews and Meta-Analyses for Protocols guidelines. We will search eligible studies from four main databases including MEDLINE, Web of Science, Cochrane Library and Embase. Randomised controlled trials (RCTs) and observational studies published before 10 ctober 2020 will be included. Two reviewers in our team will conduct the study selection and data extraction independently. Risk of bias will be assessed by Cochrane Collaboration criteria and the Newcastle-Ottawa scale for RCTs and observational studies, respectively. We will assess the good functional outcomes defining the modified Rankin scale score $\leq 2$ at 90 days after treatment, short-term stroke severity as National Institutes of Health Stroke Scale score at 24 hours after intervention, and successful recanalisation as a modified Thrombolysis in Cerebral Infarction scale score of $\geq 2 \mathrm{~b}$ after intervention. Also, safety outcomes will be assessed. The performance of this meta-analysis will depend on the quantity of included studies. The assessment of study heterogeneity will be performed by the $I^{2}$ statistic. If there is mild heterogeneity $\left(I^{2}<20 \%\right)$ of intervention outcomes in included studies, the fixed-effect model will be applied; otherwise, the random-effect model will be performed. Subgroup analyses and an assessment of publication bias will also be conducted with sufficient data.

Ethics and dissemination No collection of primary data from patients is needed. Therefore, the ethical approval is unnecessary. The results may be presented in a peerreviewed journal and related conferences.

PROSPERO registration number CRD42020176764.
Strengths and limitations of this study

- This systematic review and meta-analysis will update the existing clinical evidence of treatment and patient selection for acute basilar artery occlusion.

- Observational studies may be included in order to obtain adequate statistics to effectively evaluate the outcomes of standard medical treatment (SMT) and SMT plus endovascular thrombectomy.

- Subgroup and sensitivity analyses will be conducted if the heterogeneity is high.

- The included observational studies will introduce risk of bias, but our assessments and methods will be meticulous to ensure the accuracy of our results.

\section{INTRODUCTION}

Stroke is closely related with morbidity and mortality. ${ }^{12}$ Nearly $10 \%$ of strokes are caused by large artery disease in the posterior circulation, especially acute basilar artery occlusion (BAO) ${ }^{1-3}$ Although acute $\mathrm{BAO}$ accounts for only $5 \%-10 \%$ of all proximal intracranial occlusions, its rates of unfavourable outcomes and fatality are $70 \%-90 \%{ }^{1-6}$ Therefore, the selection of optimal treatments for these patients is necessary to obtain the timely and successful revascularisations needed to improve their clinical outcomes.

The two predominant treatment strategies for $\mathrm{BAO}$ are standard medical treatment (SMT) and SMT plus endovascular thrombectomy (EVT), and the superiority of these two methods has been debated. ${ }^{3}{ }^{7-9}$ SMT includes intravenous thrombolysis, systematic anti-coagulation, antiplatelet medications or combinations of these medical treatments. ${ }^{8}$ SMT plus EVT includes stent retrieval thrombectomy, thromboaspiration, stenting, intraarterial thrombolysis, balloon angioplasty or combinations of any of these approaches. ${ }^{389}$ 
The reports of many randomised controlled trials (RCTs) have concluded that SMT plus EVT is superior to SMT alone for patients with proximal anterior circulation stroke. ${ }^{10-12}$ However, the optimal treatment for acute BAO still remains controversial. One previous prospective registry study, the Basilar Artery International Cooperation Study, was conducted more than 10 years ago before modern EVT techniques and mechanical recanalisation devices were available. ${ }^{7}$ Therefore, the results may not be applicable to current practice. In addition, two recently published clinical trials, including the Endovascular Treatment versus Standard Medical Treatment for Vertebrobasilar Artery Occlusion ${ }^{3}$ and the EVT for Acute Basilar Artery Occlusion Study (the European Stroke Organisation and the World Stroke Organization in May 2020) report different results. Furthermore, although previous meta-analyses have reported that SMT plus EVT had superior outcomes and better recanalisation rates than those of SMT alone in patients with acute $\mathrm{BAO},{ }^{12413}$ the pooled data were only from observational studies with a single-arm nature and small sample size. ${ }^{48}$ Thus, updating the existing clinical evidence to ensure effective treatment selection in patients with acute $\mathrm{BAO}$ is necessary.

Therefore, we will systemically review and summarise literatures to evaluate current treatment modalities of SMT with SMT plus EVT, aiming to provide updated and reliable evidence for clinicians and health decisionmakers when treating acute $\mathrm{BAO}$.

\section{METHODS AND ANALYSIS}

This protocol is drafted referring to the Preferred Reporting Items for Systematic Reviews and Meta-Analyses for Protocols (PRISMA-P) guidelines ${ }^{1415}$ (online supplemental file 1, PRISMA-P checklist). The registration of this systematic review and meta-analysis is shown on the International Prospective Register of Systematic Reviews 'PROSPERO' database. If any modification is made to this protocol, the record will be updated in PROSPERO.

\section{Search strategy}

A literature search will be performed by two independent reviewers. We will search MEDLINE, Web of Science, the Cochrane Library and Embase. We will also search WHO trial register (https://www.who.int/ictrp/en/) for potential studies. Database-specific, controlled vocabulary and additional free-text terms for the concepts of 'BAO', 'vertebrobasilar occlusion', 'acute ischaemic stroke', 'endovascular treatment', 'thrombectomy', 'medical treatment', 'posterior circulation' and 'occlusion' will be used to capture all possible eligible studies. The searches will be restricted to studies published in English before 1 October 2020. The search terms will be provided in detail as online supplementary material (online supplemental file 2, Search strategy).

\section{Study selection}

The study selection will strictly follow criteria from the Population, Intervention, Comparison, Outcome model.

\section{Eligibility criteria}

1. Patient

Patients aged 18 years or older with acute ischaemic stroke caused by $\mathrm{BAO}$ or flow deprivation to the basilar artery (eg, functional $\mathrm{BAO}$ ) due to occlusion of the distal intracranial vertebral artery (V4 segment) will be included. Arterial occlusion will be confirmed by imaging studies, such as CT angiography or digital subtraction angiography. In addition, the modified Rankin scale (mRS) score should be $\leq 2$ before intervention. Related anatomy, clinical symptoms and diagnosis methods of the posterior circulation stroke have been illustrated by previous researches and will be referred to when we conduct this systemic review and metaanalysis. ${ }^{1617}$

2. Intervention

The intervention will be SMT plus EVT and include stent retrieval thrombectomy, thromboaspiration, stenting, intra-arterial thrombolysis, balloon angioplasty or combinations of any of these approaches. EVT should be initiated within 24 hours of the estimated time of BAO.

3. Comparator

The comparator will be SMT alone, which includes intravenous thrombolysis (recombinant tissue plasminogen activator or urokinase), systematic anti-coagulation, antiplatelet medications or combinations of these medical treatments.

4. Outcomes

At least one of the following items should be reported: (a) Efficacy

- Primary efficacy outcome: the $\mathrm{mRS} \leq 2$ at 90 days after intervention as good functional outcome.

- Secondary efficacy outcomes: National Institutes of Health Stroke Scale (NIHSS) score at 24 hours after intervention as short-term stroke severity, and the modified Thrombolysis in Cerebral Infarction scale $\geq 2 \mathrm{~b}$ after intervention as successful recanalisation.

(b) Safety

- Mortality $(m R S=6)$ at 90 days after intervention.

- Other outcomes, such as symptomatic intracranial haemorrhage and other severe adverse events, such as pneumonia, heart failure and systemic haemorrhage. The definition of symptomatic intracranial haemorrhage is intracranial haemorrhage based on imaging and $\geq 4$ points increase of the NIHSS score within 24hours after intervention.

5. Study type

- RCTs and observational studies (cohort studies, casecontrol studies) will be included. The inclusion of observational studies is to gather sufficient data for outcome evaluation and to minimise the type II errors that can result from the lack of statistical power found in sole RCTs. ${ }^{1819}$ 


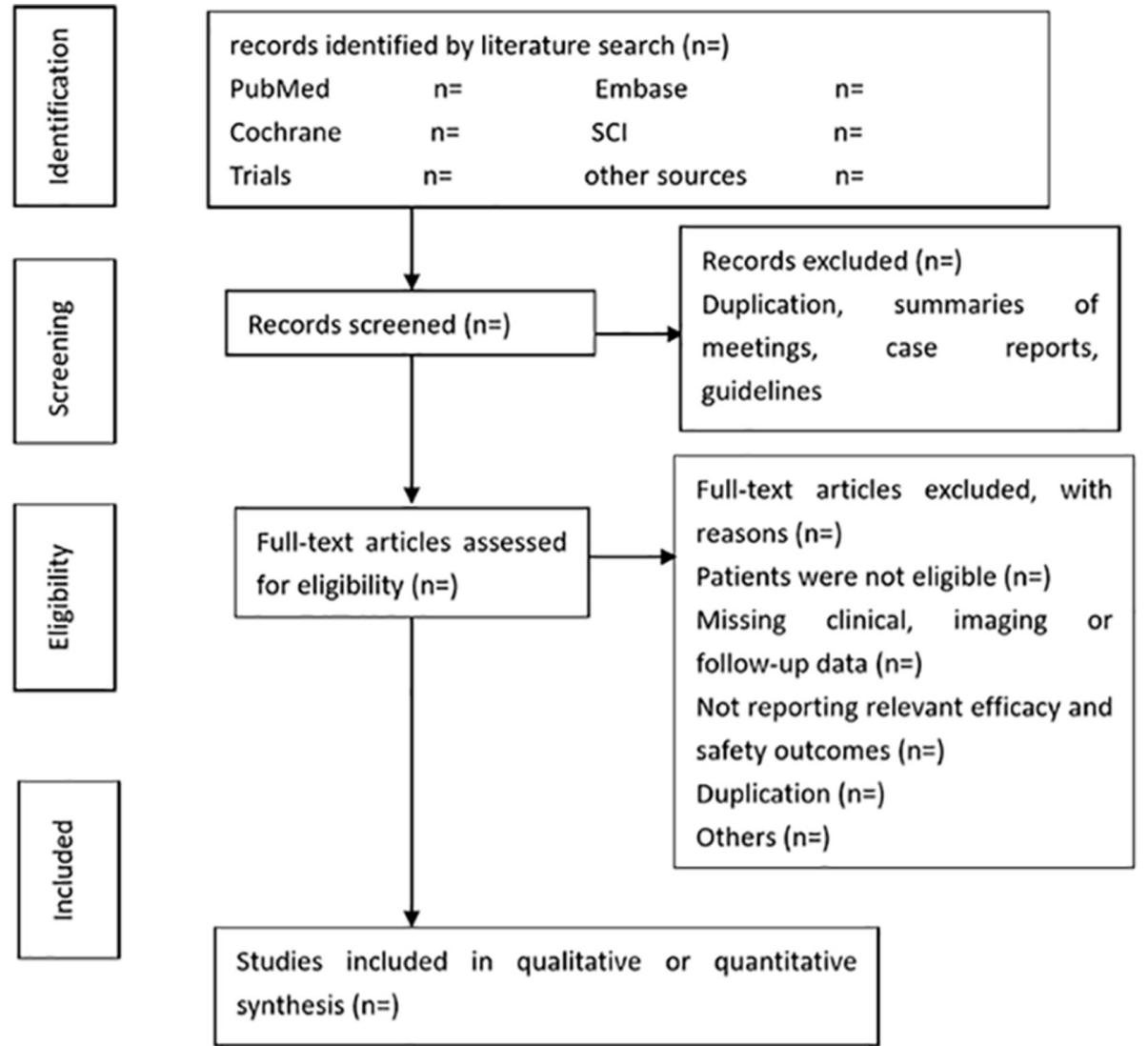

Figure 1 Flow diagram of literature for systematic review and meta-analysis.

\section{Exclusion criteria}

1. Patients with intracranial haemorrhage, significant cerebellar mass effect and acute hydrocephalus on CT or MRI before stroke.

2. Inability to extract separate data from SMT or EVT treatment.

3. No report about aforementioned outcomes or an inability to extract the exact number of complications.

4. Unsuitable study types, such as case report, case series, studies with a sample size less than 10 and studies with unavailable full text.

\section{Data selection and analysis}

\section{Selection of studies}

Two reviewers will screen and select the eligible studies, independently (figure 1). First, the reviewers will screen titles, keywords and abstracts and then exclude irrelevant studies. Second, we will acquire the full articles of all remaining studies. Subsequently, the reviewers will read the studies to assess their eligibility for inclusion and document the reasons for study exclusion. When data from the same trial are reported in more than one article, we will select the most recent study or the one with the largest sample size. A third reviewer will help to solve any disagreements between the former two reviewers.

\section{Data collection}

A standardised form will be used for data extraction by two reviewers independently. The extracted data will include the following information:
1. Study characteristics, such as type of study, authors, year of publication, sample size and number of patients.

2. Patient characteristics, such as mean age, gender, medical history, site of occlusion and baseline NIHSS score.

3. Intervention characteristics, such as the type of endovascular treatment and medical treatment.

4. Efficacy and safety outcomes.

A third reviewer will be involved when a disagreement cannot be resolved by discussion. If an included study has unclear or missing data, we will contact the corresponding authors.

\section{Risk of bias assessment}

Risk of bias exists in included studies, and two independent reviewers will evaluate it. The Cochrane Collaboration criteria (RoB tool V.2) and the Newcastle-Ottawa scale will be used for RCTs and observational studies (online supplemental file 3). ${ }^{18}{ }^{19}$ Each domain of included studies will be given a score on the risk of bias.

\section{Measures of treatment effect and data synthesis}

Treatment effect will be reported as relative risk with $95 \%$ CIs for the outcomes of dichotomous data or categorical data. For continuous variables, such as NIHSS score, we will use mean differences with $95 \%$ CIs. Only in the case of there being sufficient sample size in three or more studies, we will conduct a meta-analysis of outcomes; a narrative presentation of the study results will be provided under circumstance of lacking data. ${ }^{19}$ For interventional 
data from RCTs and observational data from observational studies, we will combine these data. The software RevMan V.5.3 will be used to analyse all data.

\section{Assessment of clinical and methodological heterogeneity Assessment of heterogeneity}

Heterogeneity will be measured with the $\mathrm{I}^{2}$ statistic before any outcome is pooled. The results will be classified into mild $(<40 \%)$, moderate $(40 \%-60 \%)$ or substantial $(>60 \%)$. If there is substantial heterogeneity and a sufficient number of included trials, we will use subgroup analyses to examine the possible origins of heterogeneity, which may include different study types, locations, patient characteristics and endovascular treatments. Sensitivity analyses will also be conducted to evaluate the effect of exclusion for the study with a high overall risk bias.

\section{Assessment of publication biases}

We will conduct a thorough protocol review of the included studies to evaluate reporting biases. As the number of included studies will possibly go beyond 10 , funnel plot is suitable for assessing publication bias.

\section{Assessment of pooled effect estimates}

If there is mild heterogeneity $\left(\mathrm{I}^{2}<20 \%\right)$ of intervention outcomes in included studies, the Mantel-Haenszel method of fixed-effect model will be applied; otherwise, the DerSimonian and Laird method of random effects model will be performed. ${ }^{20}$ The standard of statistical significance is $p$ value $<0.05$. When statistical pooling is determined to be unrealistic because of substantial heterogeneity, the results will be presented in tables and discussed afterwards.

The guideline of the Grading of Recommendations Assessment, Development and Evaluation system will be used to evaluate the quality of evidence that contributes to the pooled-effect estimates of the main outcomes of RCTs or the quality of evidence of single study. We will then construct a table that summarises the overall study results.

\section{Patient and public involvement}

As the present study is a systematic review based on published data, patient and public are not involved in the study design, conduct, data analysis and result dissemination.

\section{DISCUSSION}

Although SMT plus EVT has been reported to be superior to SMT alone in many previous RCTs at improving proximal anterior circulation in patients with stroke, ${ }^{21-25}$ the safety and efficacy of EVT to treat patients of acute BAO remains uncertain. Therefore, an updated and high quality systematic review and meta-analysis for acute BAO treatment is needed. We expect that this study will provide direct and practical clinical evidence for clinicians who must decide, which treatment is suitable for acute BAO, as well as guide future research. Thus, patients with acute $\mathrm{BAO}$ could obtain maximal benefit from the first therapeutic choice recommended from current guidelines.

\section{Ethics and dissemination}

Ethical approval for this systematic review and meta-analysis is not needed because we will not collect the primary data of patients. We will publish and present the results of this study in a peer-reviewed journal and related conferences.

\section{Author affiliations}

${ }^{1}$ Department of Neurosurgery, Xuanwu Hospital, Capital Medical University, Beijing, China

${ }^{2}$ China International Neuroscience Institute (China-INI), Beijing, China

${ }^{3}$ Department of Medical Imaging, University of Toronto Faculty of Medicine, Toronto, Ontario, Canada

${ }^{4}$ Neuroradiology \& Neurointervention Service, Brigham and Women's Hospital, Harvard Medical School, Boston, Massachusetts, USA

${ }^{5}$ Department of Evidence-based Medicine, Xuanwu Hospital, Capital Medical University, Beijing, China

${ }^{6}$ Medical Library, Xuanwu Hospital, Capital Medical University, Beijing, China ${ }^{7}$ Department of Interventional Neuroradiology, Xuanwu Hospital, Capital Medical University, Beijing, China

Acknowledgements We would like to thank Editage (www.editage.cn) for English language editing.

Contributors XB, TW, LJ and YM contributed to the initial idea for this study. XW, $X Z$ and $Y F$ developed and revised the search strategy. XB, LL, TW and XZ completed the study design. $\mathrm{LJ}$ and $\mathrm{YM}$ contributed to consults about clinical issues. $\mathrm{XB}, \mathrm{XZ}, \mathrm{LL}$ and $A A D$ contributed to the original draft. $L J, Y M$ and $K Y$ contributed to the revision of the draft. XB, XZ and LL contributed equally to this article. All of the authors approved the final work prior to submission.

Funding This work was supported by the Ministry of Science and Technology of the People's Republic of China (2016YFC1301700) and the Beijing Scientific and Technologic Project (Z201100005520019).

Competing interests None declared.

Patient consent for publication Not required.

Ethics approval Not required.

Provenance and peer review Not commissioned; externally peer-reviewed.

Supplemental material This content has been supplied by the author(s). It has not been vetted by BMJ Publishing Group Limited (BMJ) and may not have been peer-reviewed. Any opinions or recommendations discussed are solely those of the author(s) and are not endorsed by BMJ. BMJ disclaims all liability and responsibility arising from any reliance placed on the content. Where the content includes any translated material, BMJ does not warrant the accuracy and reliability of the translations (including but not limited to local regulations, clinical guidelines, terminology, drug names and drug dosages), and is not responsible for any error and/or omissions arising from translation and adaptation or otherwise.

Open access This is an open access article distributed in accordance with the Creative Commons Attribution Non Commercial (CC BY-NC 4.0) license, which permits others to distribute, remix, adapt, build upon this work non-commercially, and license their derivative works on different terms, provided the original work is properly cited, appropriate credit is given, any changes made indicated, and the use is non-commercial. See: http://creativecommons.org/licenses/by-nc/4.0/.

ORCID iDs

Tao Wang http://orcid.org/0000-0003-1225-0173

Adam Andrew Dmytriw http://orcid.org/0000-0003-0131-5699

Yao Feng http://orcid.org/0000-0001-7923-8158

Liqun Jiao http://orcid.org/0000-0003-4982-6295

\section{REFERENCES}

1 Wyszomirski A, Szczyrba S, Tomaka D, et al. Treatment of acute basilar artery occlusion: systematic review and meta-analysis. Neurol Neurochir Pol 2017;51:486-96.

2 Kumar G, Shahripour RB, Alexandrov AV. Recanalization of acute basilar artery occlusion improves outcomes: a meta-analysis. $J$ Neurointerv Surg 2015;7:868-74.

3 Liu X, Dai Q, Ye R, et al. Endovascular treatment versus standard medical treatment for vertebrobasilar artery occlusion (BEST): 
an open-label, randomised controlled trial. Lancet Neurol 2020;19:115-22.

4 Sheng K, Tong M. Therapy for acute basilar artery occlusion: a systematic review and meta-analysis. F1000Res 2019;8:165.

5 Chiang C-C, Dumitrascu OM, Wingerchuk DM, et al. Acute basilar artery occlusion: does recanalization improve clinical outcome? A critically Appraised topic. Neurologist 2018;23:71-4.

6 Singer OC, Berkefeld J, Nolte $\mathrm{CH}$, et al. Mechanical recanalization in basilar artery occlusion: the ENDOSTROKE study. Ann Neurol 2015;77:415-24.

7 Schonewille WJ, Wijman CAC, Michel P, et al. Treatment and outcomes of acute basilar artery occlusion in the basilar artery international cooperation study (BASICS): a prospective registry study. Lancet Neurol 2009;8:724-30.

8 Writing Group for the BASILAR Group, Zi W, Qiu Z, et al. Assessment of endovascular treatment for acute basilar artery occlusion via a nationwide prospective registry. JAMA Neurol 2020;77:561-73.

9 Lindsberg PJ, Mattle HP. Therapy of basilar artery occlusion: a systematic analysis comparing intra-arterial and intravenous thrombolysis. Stroke 2006;37:922-8.

10 Lin Y, Schulze V, Brockmeyer M, et al. Endovascular thrombectomy as a means to improve survival in acute ischemic stroke: a metaanalysis. JAMA Neurol 2019;76:850.

11 Campbell BCV, van Zwam WH, Goyal M, et al. Effect of general anaesthesia on functional outcome in patients with anterior circulation ischaemic stroke having endovascular thrombectomy versus standard care: a meta-analysis of individual patient data. Lancet Neurol 2018;17:47-53.

12 Turk AS, Siddiqui A, Fifi JT, et al. Aspiration thrombectomy versus stent retriever thrombectomy as first-line approach for large vessel occlusion (COMPASS): a multicentre, randomised open label, blinded outcome, non-inferiority trial. The Lancet 2019;393:998-1008.

13 Shu L, Salehi Ravesh M, Jansen O, et al. Stent retriever thrombectomy potentially increases the recanalization rate, improves clinical outcome, and decreases mortality in acute basilar occlusion: a systematic review and meta-analysis. Cerebrovasc Dis Extra 2019;9:46-56.
14 Shamseer L, Moher D, Clarke M, et al. Preferred reporting items for systematic review and meta-analysis protocols (PRISMA-P) 2015: elaboration and explanation. BMJ 2015;349:97647.

15 Hutton B, Salanti G, Caldwell DM, et al. The PRISMA extension statement for reporting of systematic reviews incorporating network meta-analyses of health care interventions: checklist and explanations. Ann Intern Med 2015;162:777-84.

16 Sparaco M, Ciolli L, Zini A. Posterior circulation ischaemic stroke-a review Part I: anatomy, aetiology and clinical presentations. Neurol Sci 2019;40:1995-2006.

17 Markus HS, van der Worp HB, Rothwell PM. Posterior circulation ischaemic stroke and transient ischaemic attack: diagnosis, investigation, and secondary prevention. Lancet Neurol 2013;12:989-98.

18 Bai X, Feng Y, Yang K, et al. Extracranial-Intracranial bypass surgery for occlusive atherosclerotic disease of the anterior cerebral circulation: protocol for a systematic review and meta-analysis. Syst Rev 2020:9:70.

19 Feng Y, Li L, Bai X, et al. Risk factors for new ischaemic cerebral lesions after carotid artery stenting: protocol for a systematic review and meta-analysis. BMJ Open 2019;9:e030025.

20 Cheung MW-L, Vijayakumar R. A guide to conducting a metaanalysis. Neuropsychol Rev 2016;26:121-8.

21 Goyal M, Demchuk AM, Menon BK, et al. Randomized assessment of rapid endovascular treatment of ischemic stroke. $N$ Engl J Med 2015;372:1019-30.

22 Campbell BCV, Mitchell PJ, Kleinig TJ, et al. Endovascular therapy for ischemic stroke with perfusion-imaging selection. N Engl J Med 2015;372:1009-18.

23 Berkhemer OA, Fransen PSS, Beumer D, et al. A randomized tria of intraarterial treatment for acute ischemic stroke. $N$ Engl $\mathrm{J}$ Med 2015;372:11-20.

24 Jovin TG, Chamorro A, Cobo E, et al. Thrombectomy within 8 hours after symptom onset in ischemic stroke. $N$ Engl $J$ Med 2015;372:2296-306.

25 Saver JL, Goyal M, Bonafe A, et al. Stent-retriever thrombectomy after intravenous t-PA vs. t-PA alone in stroke. N Engl J Med 2015;372:2285-95. 